\title{
Optical parametric generation in CGA crystal
}

\author{
K.L. Vodopyanov ${ }^{\mathrm{a}, *}$, G.M.H. Knippels ${ }^{\mathrm{b}}$, A.F.G. van der Meer $^{\mathrm{b}}$, \\ J.P. Maffetone ${ }^{\mathrm{c}}$, I. Zwieback ${ }^{\mathrm{c}}$ \\ a Informed Diagnostics, Inc., 1050 E. Duane Ave., suite H, Sunnyvale, CA 94085, USA \\ b FOM-Inst. for Plasma Physics, 'Rijnhuizen', Edisonbaan 14, 3439 MN Nieuwegein, Netherlands \\ ${ }^{\mathrm{c}}$ Inrad, Inc., 181 Legrand Ave., Northvale, NJ 07647, USA
}

Received 17 July 2001; received in revised form 6 September 2001; accepted 15 November 2001

\begin{abstract}
We have demonstrated optical parametric generation (OPG) in CGA crystal known for its extremely high secondorder nonlinearity $(236 \mathrm{pm} / \mathrm{V})$. A single-pass OPG was pumped by $10 \mu \mathrm{J}, 600 \mathrm{fs}$ pulses at $\lambda=5 \mu \mathrm{m}$ and the output was tunable in the 7-18 $\mu \mathrm{m}$ range. Efficient second harmonic generation of 9-11 $\mu \mathrm{m}$ radiation with an internal conversion efficiency of $62 \%$ has also been demonstrated. (c) 2002 Elsevier Science B.V. All rights reserved.
\end{abstract}

$\mathrm{CdGeAs}_{2}$ (CGA) crystal is known for its highest second-order nonlinearity $\left(d_{\text {eff }}=236 \mathrm{pm} / \mathrm{V}\right)$ among any crystals in practical use (for example, its nonlinear-optical figure of merit, $d_{\mathrm{eff}} / n^{3}$, is 42 times larger than that of periodically poled lithium niobate). It transmits light between 2.4 and $18 \mu \mathrm{m}$ and is a perfect candidate for second-harmonic generation (SHG) and nonlinear-optical frequency conversion via optical parametric oscillation (OPO), specifically into the second atmospheric window $(8-12 \mu \mathrm{m})$. In the past, the linear absorption of as-grown CGA crystals was high $\left(\sim 1 \mathrm{~cm}^{-1}\right)$ which precluded its wide use in nonlinear optics.

\footnotetext{
${ }^{*}$ Corresponding author. Fax: +1-408-524-0552.

E-mail addresses: kostya@infodiag.com, kvodopyanov@, infodiag.com (K.L. Vodopyanov).
}

Lately, it became possible, owing to the advancements in the crystal growth and after-growth processing, to produce large size CGA crystals with improved transmission [1-3]. As a result, highly efficient frequency doubling of $\mathrm{CO}_{2}$ laser radiation (conversion efficiency $28 \%$ at room temperature and $50 \%$ at $77 \mathrm{~K}$ ) [1] and difference frequency generation (at room temperature) with photon conversion efficiency $>20 \%$ in the $7-20 \mu \mathrm{m}$ spectral range [3] have been reported in CGA. Despite the fact that pulsed thresholds as low as 30 $\mu \mathrm{J}$ are expected in a singly resonant CGA OPO, the great potential of this crystal as an OPO/OPG material has not been materialized so far. In this paper, we report the first demonstration of optical parametric generation (OPG) in CGA (which we regard as a logical step to achieving an OPO in this crystal), as well as efficient SHG operation. 


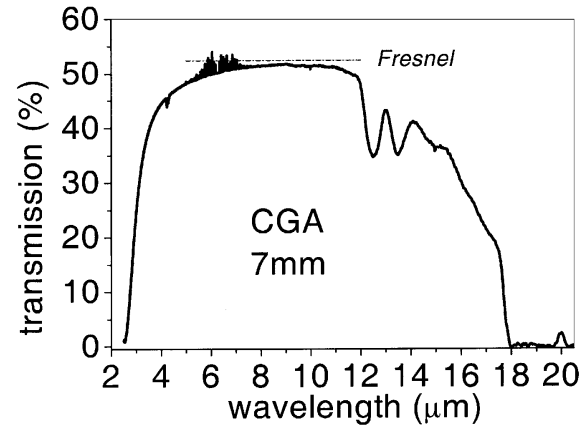

Fig. 1. Measured transmission spectrum of the uncoated CGA crystal. Dash-dotted curve corresponds to Fresnel reflection losses (refractive index $n \approx 3.5$ ).

The CGA crystal was grown at Inrad, by the horizontal gradient freeze technique, and was subjected to post-growth treatment (irradiation and annealing) [2] to decrease its room temperature optical absorption. The $6 \times 8 \mathrm{~mm}^{2}$ cross-section, $7 \mathrm{~mm}$ long sample designed for type I phase matching was cut at $\theta=33^{\circ}$ and $\varphi=0^{\circ}$. The crystal faces were polished but not anti-reflection coated. Fig. 1 shows the measured transmission spectrum. The values of room-temperature absorption coefficient of less than $0.1 \mathrm{~cm}^{-1}$ were achieved in the whole range $\lambda=5-12 \mu \mathrm{m}$. This is comparable to the results of Schunemann et al. [4] reported recently.

As a pump source for the OPG, we have used $\lambda \approx 5 \mu \mathrm{m}$ pulses from the Free-Electron Laser FELIX in Nieuwegein, Netherlands [5]. Schematic of the OPG experiment is shown in Fig. 2.
The laser beam consisted of a train of short pulses (micro-pulses), which are separated by intervals of $40 \mathrm{~ns}$. The duration of the train (macropulse) was approximately $4 \mu \mathrm{s} \quad(\sim 100$ micropulses) with the total energy $\sim 1 \mathrm{~mJ}$ and the overall laser repetition rate was $10 \mathrm{~Hz}$. The laser micro-pulse duration was $\tau \approx 600$ fs at a FWHM spectral width of $24 \mathrm{~cm}^{-1}$. (It has been found in the previous experiments [6], using autocorrection technique with a GaSe crystal that the timebandwidth product for the FELIX pulses is close to 0.44 , i.e. near the value for the Fourier-transform-limited Gaussian pulses.) The pump laser beam was focused into the CGA crystal using a telescope made of two $\mathrm{BaF}_{2}$ lenses with $f=+15$ and $f=-5 \mathrm{~cm}$ and had a beam size $\left(1 / e^{2}\right.$ intensity radius) of $w_{0} \approx 450 \mu \mathrm{m}$ (which is larger than the birefringent walk-off in the CGA crystal: $160 \mu \mathrm{m}$ at $L=7 \mathrm{~mm}$ and $\theta=33^{\circ}$ ). The OPG output was monitored using a long-pass $(\lambda>5.5 \mu \mathrm{m})$ filter and MCT $(77 \mathrm{~K})$ or pyroelectric detector. An OPG threshold was observed at the incoming peak laser intensity of approximately $I_{\mathrm{L}}=3 \mathrm{GW} / \mathrm{cm}^{2}$. This is close to the calculated value $\left(2.2 \mathrm{GW} / \mathrm{cm}^{2}\right)$ [7] obtained from our numerical modeling of the three-wave interaction process. The modeling assumed Gaussian pulse shape in time and transverse coordinate and neglected group velocity mismatch between the three interacting waves (the time walk-off between any of the three beams was less than 55 fs at $7 \mathrm{~mm}$ crystal length, which is much shorter than the laser pulse duration).

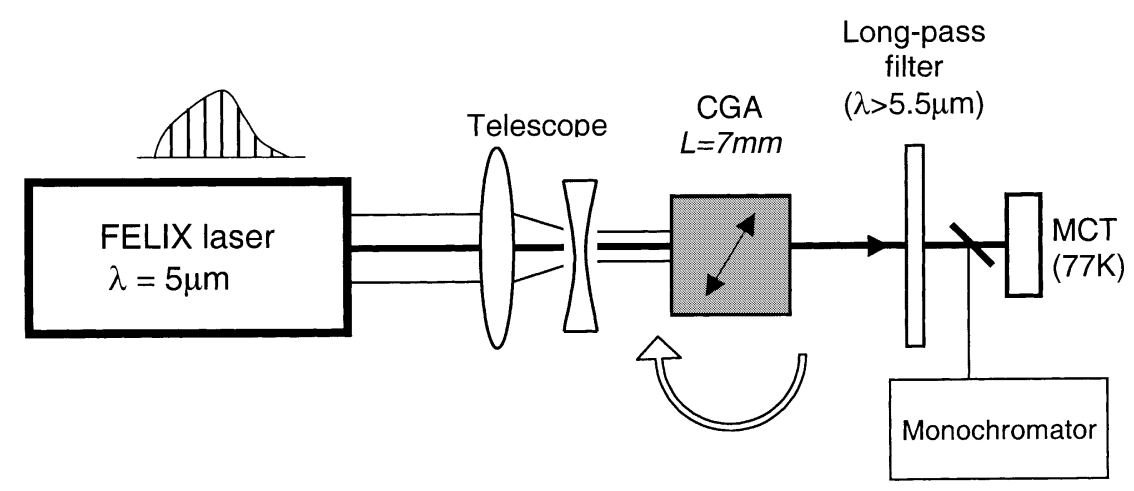

Fig. 2. Schematic of the OPG experiment. 
The OPG output was easily detectable when it was angular-tuned within the range $7-18 \mu \mathrm{m}$. The experimental OPG angular tuning curve is shown in Fig. 3. The $n_{\mathrm{o}}$ and $n_{\mathrm{e}}$ dispersion relations of Kato [8], provide the best agreement between the calculated curve (Fig. 3, dash-dotted line) and experimental tuning data. The spectral width of the OPG output (measured at the signal wave, $\lambda_{\mathrm{s}}$, with a TRIAX 550 Jobin Yvon monochromator) was typically $250 \mathrm{~cm}^{-1}$-away from degeneracy $\left(\lambda_{\mathrm{s}} \sim 8 \mu \mathrm{m}, \lambda_{\mathrm{i}} \sim 13 \mu \mathrm{m}\right)$, and $600 \mathrm{~cm}^{-1}$-near degeneracy at $\lambda_{\mathrm{s}} \sim \lambda_{\mathrm{i}} \sim 10 \mu \mathrm{m}$. These values are on the same order as the calculated OPG acceptance bandwidth for the $7 \mathrm{~mm}$ CGA crystal: $150 \mathrm{~cm}^{-1}$ (away from degeneracy) and $590 \mathrm{~cm}^{-1}$ (at the degeneracy).

Fig. 4 shows the FELIX laser macro-pulse envelope, along with the OPG pulse envelope, both recorded with slow ( $\sim 100 \mathrm{~ns})$ integrating detectors. The width of the OPG pulse envelope is much narrower since the OPG conversion efficiency is strongly dependent on peak power. At the peak pump intensity of $I_{\mathrm{L}}=6 \mathrm{GW} / \mathrm{cm}^{2}$, and typical OPG internal photon conversion efficiency was $3 \%$.

Taking advantage of the broad tunability of the FELIX laser, we have also performed type-I, SHG experiments in the range of fundamental wavelengths $9-11 \mu \mathrm{m}$. In, this case, we have used FELIX

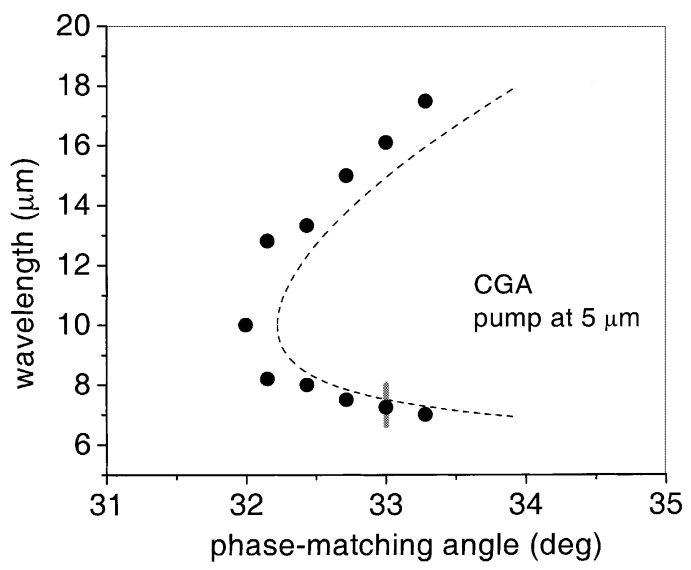

Fig. 3. Angular tuning curves of type-I OPG pumped at $\lambda=5 \mu \mathrm{m}$. Vertical bar corresponds to the typical FWHM linewidth. Dashed line - calculated from known dispersion relations [8].

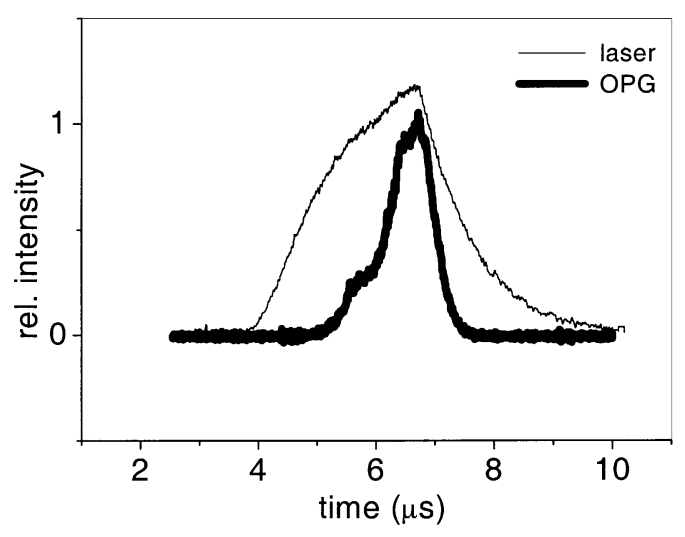

Fig. 4. Pump laser macro-pulse envelope (thin line) and the OPG pulse envelope (thick line).

pulses with $\tau=3.9$ ps duration $\left(\Delta v=3.8 \mathrm{~cm}^{-1}\right)$ focused to a beam size of $w_{0} \approx 400 \mu \mathrm{m}$. The inset to Fig. 5 represents the SHG phase-matching angular curve $10 \mu \mathrm{m} \rightarrow 5 \mu \mathrm{m}$, which is peaked, at $\theta=32.0^{\circ}$. This was used to get the missing data point in the OPG tuning curve of Fig. 3 at exactly the OPG degeneracy. SHG phase-matching angles for other wavelengths were found to be $\theta=32.2^{\circ}$ $\left(\lambda_{\omega}=9 \mu \mathrm{m}\right)$, and $\theta=32.46^{\circ}\left(\lambda_{\omega}=11 \mu \mathrm{m}\right)$. Fig. 5 represents internal SHG conversion efficiency $10 \mu \mathrm{m} \rightarrow 5 \mu \mathrm{m}$ as a function of the incident peak intensity, from which one can see that an efficiency as high as $62 \%$ can be reached at $380 \mathrm{MW} / \mathrm{cm}^{2}$.

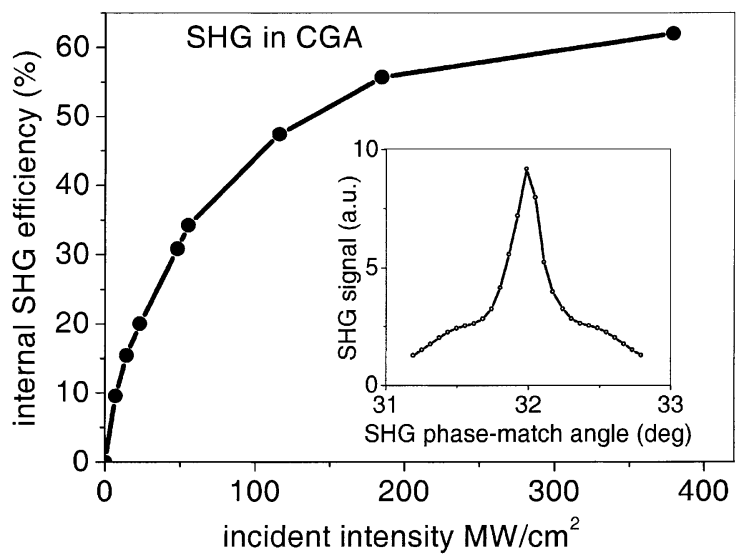

Fig. 5. SHG $(10 \mu \mathrm{m} \rightarrow 5 \mu \mathrm{m})$ internal conversion efficiency in CGA, as a function of the incident peak laser intensity. Inset: the SHG phase-matching angular curve. 
In conclusion, we have demonstrated the first CGA-based OPG which was pumped by subpicosecond pulses at $5 \mu \mathrm{m}$ and which was continuously tunable between 7 and $18 \mu \mathrm{m}$. We regard this as a step to achieving a, low-threshold OPO pumped by nanosecond pulses in this crystal. In particular, we have measured experimental OPG/ OPO angular tuning curves for CGA, in a large range of wave lengths, which is essential for future OPO designs. We have also demonstrated efficient SHG, with a $62 \%$ internal conversion efficiency, using 3.9 ps pulses in, the $9-11 \mu \mathrm{m}$ range.

\section{Acknowledgements}

We are grateful to Maria Murray for the help in fabrication of CGA crystals. The experiment at the FELIX laser was part of the research program of the "Stichting voor fundamenteel Onderzoek der Materie (FOM)," and was financially supported by the "Nederlandse Organisatie voor Wetenschappelijk Onderzoek (NWO)".

\section{References}

[1] P.G. Schunemann, in: Conference on Lasers and ElectroOptics, vol. 9, 1996 OSA Technical Digest, Optical Society of America, Washington, DC, 1996, p. 230.

[2] I. Zwieback, D. Perlov, J.P. Maffetone, W. Ruderman, Appl. Phys. Lett. 73 (1998) 2185.

[3] K.L. Vodopyanov, P.G. Schunemann, Opt. Lett. 23 (1998) 1096.

[4] P.G. Schunemann, S.D. Selzler, T.M. Pollak, in: Advanced Solid State Lasers, OSA Technical Digest, Optical Society of America, Washington DC, 2001, p. 408.

[5] G.M.H. Knippels, R.F.X.A.M. Mols, A.F.G. van der Meer, D. Oepts, P.W. van Amersfoort, Phys. Rev. Lett. 75 (1995) 1755. Available from <http://www.rijnh.nl/felix/>.

[6] G.M.H. Knippels, A.F.G. van der Meer, R.F.X.A.M.P.W. Mols, P.W. van Amersfoort, R.B. Vrijen, D.J. Maas, L.D. Noordam, Opt. Commun. 118 (1995) 546.

[7] The OPG threshold in this case corresponds to the threshold of detection of the OPG signal which we assumed to be approximately $10^{-5}$ of the level of the pump power.

[8] K. Kato, Private Communications, $1997\left(n_{\mathrm{o}}^{2}=12.4008+\right.$ $2.1603 /\left(\lambda^{2}-2.0617\right)-0.00133 \cdot \lambda^{2} ; n_{\mathrm{e}}^{2}=13.0079+3.2613 /$ $\left.\left(\lambda^{2}-2.8382\right)-0.00125 \cdot \lambda^{2}\right)$. 